\title{
Developing Modular Product Family Using GeMoCURE within an SME
}

\author{
Xiu-Tian Yan $^{1 *}$ and Barry Stewart ${ }^{1}$ \\ ${ }^{1}$ Department of Design, Manufacturing and Engineering Management, The University of \\ Strathclyde, Glasgow, G1 1XJ, UK \\ *Corresponding author: Email: x.yan@strath.ac.uk,
}

\begin{abstract}
Companies adopt the strategy of producing variety of products to be competitive and responsive to market. Product variation is becoming an important factor in companies' ability to accurately meet customer requirements. Ever increasing consumer options mean that customers have more choices than ever before which put commercial pressures on companies to continue to diversify. This can be a particular problem within Small to Medium Enterprises (SMEs) who do not always have the level of resources to meet these requirements. As such, methods are required that provide means for companies to be able to produce a wide range of products at the lowest cost and shortest time. This paper details a new modular product design methodology that provides a focus on developing modular product families. The methodology's function is described and a case study detailed of how it was used within an SME to define the company's product portfolio and create a new Generic Product Function Structure from which a new family of product variants can be developed. The methodology lends itself to modular re-use which has the potential to support rapid development and configuration of product variants.
\end{abstract}

Keywords: Modules, Methodology, Mechatronics, Product Family, SME

\section{Introduction}

In today's world of high paced change and ever increasing consumer options, it is often vital for companies to diversify their product ranges to meet customers changing needs. To keep up with such strains and to help handle the subsequent complexity of the design process companies have to find new and innovative ways of managing their product development. These factors are particularly relevant within Small to Medium Enterprises (SMEs) where lack of time and resources and competitive market environments mean that contstant pressures are put on companies to grow. Modularity is a concept that is being introduced as a means to meet some of these compelxities and help in introducing a greater variety of products to the market in shorter times(Baxter et al 2007).

The heart of research into product modularity is the development of modular products, therefore, methods for developing more modular products are essential (Thyssen and Hansen, 2001). There has been much research carried out into modular design methods (Synopsys, 1999) with many different techniques and methodologies proposed to help companies create 'modular' products. The benefits of such formal tasks are well documented with reported cost savings of up to 64 times (Duffy and Ferns, 
1999) and studies showing that by implementing formal methods, as opposed to relying on designers' natural instincts, significant savings in time and resources can be achieved (Smith, 2002). One such piece of work has been detailed in previous research (Yan et al, 2007) is called the GeMoCURE methodology which proposes a modular design methodology that also takes into account product perspectives, lifecycle objectives, modular re-use and product families.

One way to meet the requirement of increasing customer requirements is to introduce product families into a company's product portfolio. A product family is generally considered to be a group of similar products that are all derived from a common product platform (Pahl and Beitz, 1994). In order to use such a concept to help companies create product variety, these platforms have to be well defined and implemented which is one of the goals of the GeMoCURE methodology. It aims to do this by creating a structure of well defined modules that can either be combined to form a product platform or added to the platform to generate new products. The methodology uses techniques that allow modules to be formed based on product functions and which takes into account the different perspectives that are inherent within any product development. Modularity is ideally suited to the concept of design for reuse i.e. reusing standard, proven components/assemblies/modules in the design of new products. This has the benefit of making a product more reliable (due to use of proven modules), cheaper due to reduced resources necessary for development (since a larger proportion of modules designed by others are used), easy to maintain, etc (Ulrich and Eppinger, 2003).

The overall objective of the design methodology is to support the creation of the greatest product variation while keeping costs, time and resources to a minimum. This paper will give a brief description of GeMoCURE methodology and examine how the methodology was implemented within a SME, how it is being used and future objectives.

\section{Modular Product Families}

The term module is used widely in many different contexts to describe a variety of different concepts. In the realm of product design, Gershenson et al (1999) state that there is no universally agreed definition for a what a module comprises of. Ulrich and Eppinger (2003) put forward the notion that ideal modules are 'chunks' of components where each 'chunk' represents one function or a series of functions. This definition is backed up by Stone et al (2000) who states that "Modules are defined as physical structures that have a one-to-one correspondence with functional structures". It is possible to summarise from these definitions and from other prominent research (Stone et al, 2003, Gershenson et al, 2003, Sosale et al, 1997, and Smith and Duffy 2001) that the main features that define a module are; structural independence, functional independence and minimal interfaces or interactions with other modules or outside influences.

The definition that has formed the foundation for the GeMoCURE methodology is built on these key points and a module is defined in this research as a set of primary product Development Primitives (PDP) which are structurally or functionally independent but are combined such that the interface between modules are standardised, interactions among PDPs within a module is encapsulated and localised and interactions between modules are concentrated and minimised.

Modularisation of products can lead to a wide range of different products but one of the uses where modularisation can be most effective is in conjunction with a common product platform. The increasingly more specific demands of customers has led to many companies introducing large product families to try and meet this wide range of needs and variety. An efficient and effective approach is to build product families based on a 


\section{GeMoCURE modular design methodology}

common product platform which allows for the accurate management of product variety (Hofer and Gruenenfelder, 2001).

Schellhammer and Karandikar (2001) define such a product platform as "...the common basis for multiple product variants targeted to meet specialised requirements for specific applications and markets." This proposes the idea that a product platform is a common base upon which modules can be added to create a wide variety of products. This type of product architecture lends itself to modular design as it allows modules to be interchanged and reused to create the maximum range of products from the components available. This is supported by Robertson and Ulrich (1998) who state that "The platform concept is characterised by the consequent modularisation of product architecture and the integration of basic (common) elements (components, functions, interfaces, design rules) over a product family."

Schellhammer and Karandikar (2001) also define the platform further by declaring that they consider the "product platform to represent a set of functions, features, parameters, components, and information around which a product architecture to base a family of products and technologies can be developed." This shows that a product platform doesn't necessarily have to consist of purely physical modules/components and can also contain the underlying technology, the product functions and or even knowledge associated with the product family.

In this study, the focused company has a goal of creating a new product family that will feature a standard product platform from which a variety of new products can be developed. In addition, the current products will be structured into well defined product families from which common modules can be found that can be stored as potential candidates for re-use in the new family.

\section{GeMoCURE Methodology}

The GeMoCURE methodology is developed as an integrated approach by combining several methods to allow designers to generate design solutions using modular concepts in a systematic manner. This new methodology contains four significant methods that form the integrated methodology; Generalisation, Modularisation, CUstomisation and REconfiguration (GeMoCURE). Figure 1 shows a detailed pictorial representation of the methodology, illustrating all detailed activities and the prescribed sequence of utilising GeMoCURE in a design and manufacturing company. The following sections detail the key process and constituent activities of the GeMoCURE methodology.

\subsection{Generalisation}

The first stage of this new methodology is called the 'Generalisation' stage and it focuses on analysing the current company product portfolio (and any new products being added) and creating generalised and generic product development primitives (PDP). This generalisation can be undertaken from two perspectives based on the work reported in (Smith, 2002, Wie et al, 2005), namely, function, and structure. Function describes the physical effect imposed on an energy, material and information flow by a design entity without regard for the working principles or physical solutions used to accomplish this effect. Structure is the most tangible concept with various approaches to partitioning physical structure into meaningful constituents such as features and interfaces in addition to the widely used assemblies and components. Additional perspectives, such as 
behaviour, solution and life-cycle can also be used to generalise modules. The output from this stage is a series of PDP models from two perspectives that provide generic artefact information and knowledge for each PDP. The methodology has been simplified slightly over previous applications to reflect the nature of the SME business and the complexity of the product portfolio.

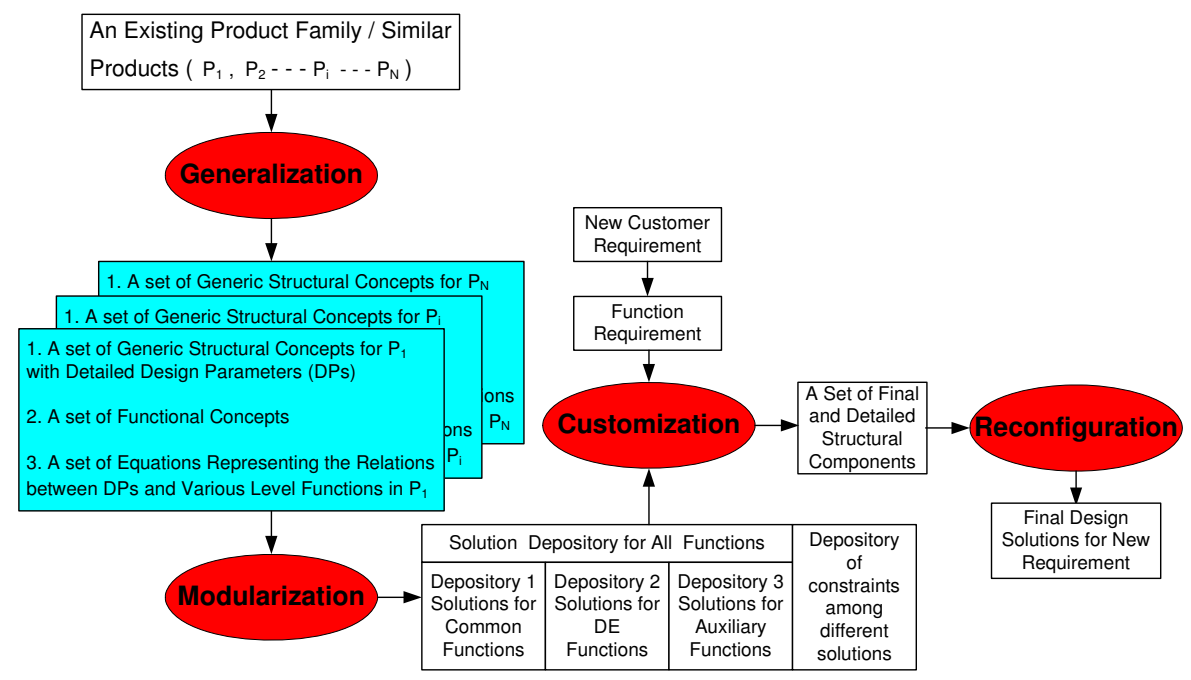

Figure 1 - The GeMoCURE design methodology brief representation

\subsection{Modularisation}

The modularisation processes are at the heart of the GeMoCURE methodology as they help to define the product families, product platforms and the derivable modules that will help to generate product variety. There are two aspects which have been considered in this approach, namely identification of generic modules and identification of distinctive modules, which focuses more on deriving modules which give unique features and characteristics for the product. The PDPs that were defined in the Generalisation stage are organised into an optimal product structure using a Dependency Structure Matrix (DSM) which uses a genetic algorithm - based on the dependencies between PDPs - to cluster the PDPs into module candidates, see Figure 2 (Smith, 2002). Based on the module definition given in section 1.2, functional modules can be identified by assessing the clusters of components using the Module Identification Module (MIM) function. This gives a visual display- see Figure 2 - of the strength of the dependencies between PDPs and allows for decisions on what makes the best module to be made.

The modules identified in Figure 2 can be assessed with a certain value called the Module Strength Indicator(MSI), which shows how strong a module is from a particular view point. Table 1 gives details of the module strength values for an industrial case study product.

Table 1 Module strength for an industrial product

\begin{tabular}{|c|c|c|}
\hline Module & $\begin{array}{c}\text { Module Strength } \\
\text { Indicator }\end{array}$ & Sub-assembly \\
\hline M1 & 0.5 & RMV Top \\
\hline M2 & 1 & Springtree \\
\hline
\end{tabular}


GeMoCURE modular design methodology

\begin{tabular}{|c|c|c|}
\hline M3 & 0.4 & Siphon \\
\hline M4 & 0.5 & RMV Bowl \\
\hline
\end{tabular}

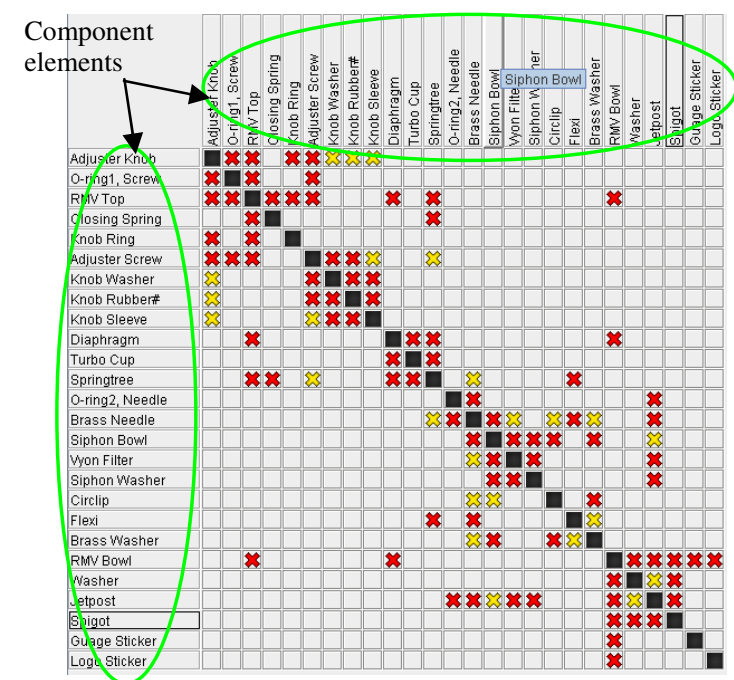

(a)

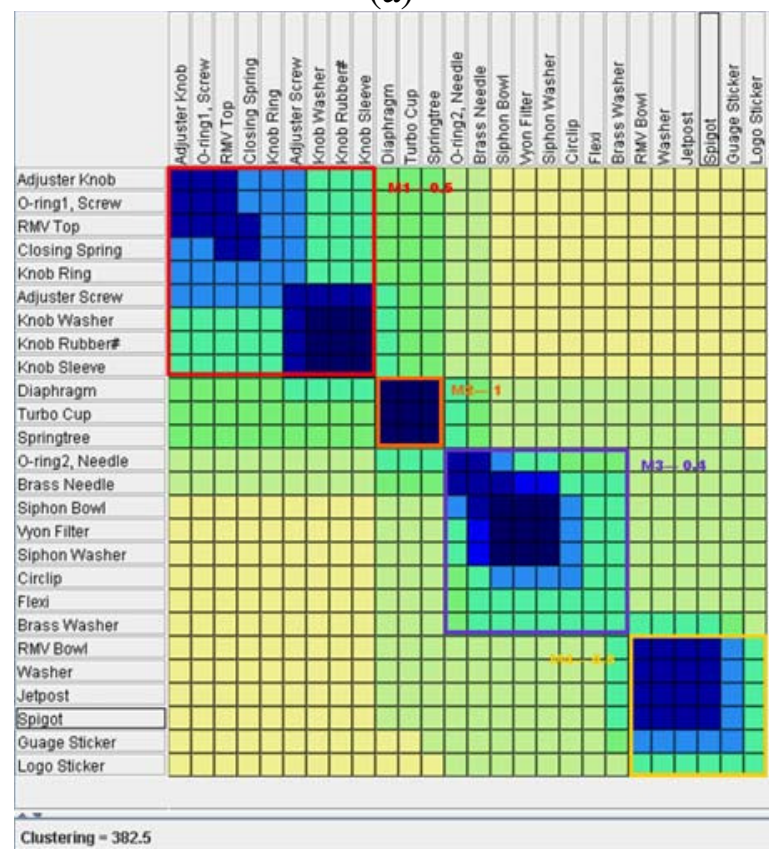

(b)

Figure 2 - (a) A Dependency Structure Matrix; (b) the Module Identification Module (MIM)

From these results the DSM is then used again to map the modular structure from the functional viewpoint to the structural viewpoint. This is called a Cross-viewpoint matrix and allows for the optimal product structure to be maintained throughout the product architecture. The structural concepts can then be stored into a solution depository where 
they can be accurately mapped onto required function and they are searchable so that new products have access to them with the option of modular re-use. The function concepts are also brought together at this point and used to define the product families - more detail on this can be found in section 1.4. Therefore the two main outputs from the generalisation stage are a depository of identified structural concepts and a well defined set of product families that describe the company's product portfolio.

\subsection{Customisation}

The Customisation stage of the process deals with the development of new products within a product family. It is a process of utilising the available modules, which were identified in the Generalisation stage, to meet a new design requirement by firstly defining the new requirement in the correct terms and then tailoring the modules in the depositories to meet the requirement. The same Generalisation and DSM techniques are used to describe the new product concept in the same terms as the product family and modules that are in the depository. By comparing the functional concepts and the solution concepts it is possible to generate solutions for the new product requirements. If there are no solutions for certain of the new product functions then these should be designed to integrate with the chosen module solutions and, once properly defined, can be added into the depository and product family.

\subsection{Reconfiguration}

Once all the modules have been selected, so that they accurately map the function structure and customer requirements, the final stage of the process is carried out. Reconfiguration takes all the modules and configures them into various product layouts or strucutre while taking into account design processes, markets, standards, interfaces, etc. During reconfiguration process, product strucntures are explored and poetntial optimal layouts will be generated. The output from this short stage will be the final product design ready for production.

Through the above customisation and reconfiguration, a family of products derived from the same modules can be generated by customise the modules to typical application scenarios and these customised and reusable products themselves become a member of the product family. Similarly, through reconfiguration it is also be able to develop new products based on the existing modules. They can form part of the product family too and this will be further illustrated in the case study section.

\section{SME Product Family Analysis}

In order to use the previous steps to create product families, the tasks highlighted in section 1.3 and in Figure 1 were carried out on an SMEs product portfolio to firstly identify the functional modules and the structural modules. To identify functions and perspective dependencies, the functional model proposed by Stone et al(2000) was created for each product variant. For the structural concepts a simple structural hierarchy was developed that showed the main structural components and their physical links. These were then added into the DSM matrix and optimised to produce optimal module structures. The functional modules were then mapped onto the structural concepts, using 
GeMoCURE modular design methodology

a cross-viewpoint matrix, to create a set of solutions for structural modules. The functional modules are then taken and analysed to assess the commonality that existed between them and their products. For each product family they are then split into three distinct categories; common functions, differentiation enable (DE) functions and auxiliary functions. Common functions describes those that are present within all product variations within a family (i.e. comprise the product platform), DE functions describe functions that are selectable and can be used to alter the performance or features of the product platform and auxiliary functions are those that don't affect he main function or product variants but provide some secondary function.

Once these have been identified they can be arranged into a schematic Generic Product Function Structure (GFPS) for the product family which shows all of the options available for product variants within that family - see Figure 3. This structure not only defines the product family but shows all the available configurations therefore opening up the possibility of rapid configuration of new product variants.

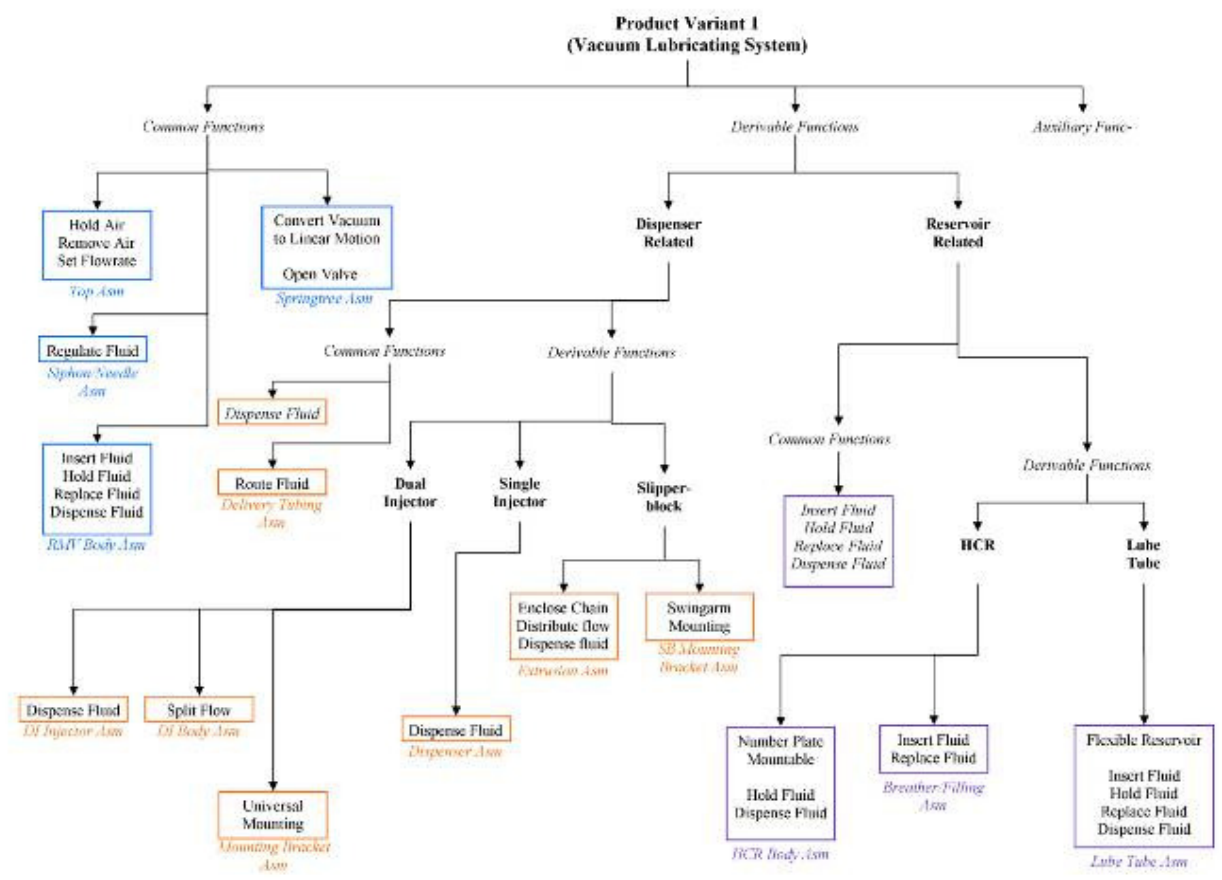

Figure 3 - An example of a Generic Product Function Structure (GPFS).from a SME

\section{Product Customisation / Configuration}

In order to maintain the product portfolio structure, new product development has to follow the steps of the methodology to enable the product to be defined in terms that will allow the product variations to be generated. When a new customer requirement is identified the first step is to carry out the Generalisation of the concepts for the new product. This will define the product in terms of its functions and allow for the inputs to be put into the DSM for the Modularisation stage. By modularising the function concepts the product can now be optimised into a modular function structure that can be used in the Customisation. 
Once the new concept has been defined in this way a function module comparison can be carried out by searching the GFPS of the product families and the Solution depository. The modules that find matches can then be allocated into the new product scheme while for any functions that don't have suitable matches a new design will have to be developed. When these new modules are developed it is necessary to keep to the optimal modular structure that was defined as closely as possible.

Once all modules - both new and re-used - have been defined it is possible to create a new GPFS for the new product family. Figure 4 shows an example of a GPFS that is constructed of both new modules and of re-used modules from other product families. By creating the product family in this manner it is possible to use the modules already used within the company in the new product family to allow for several product variants to be produced and to add to the company's overall product portfolio. The fact that so many of the modules are proven, reliable modules that are already in full scale production allows for rapid configuration of these new variants and fast time to market.

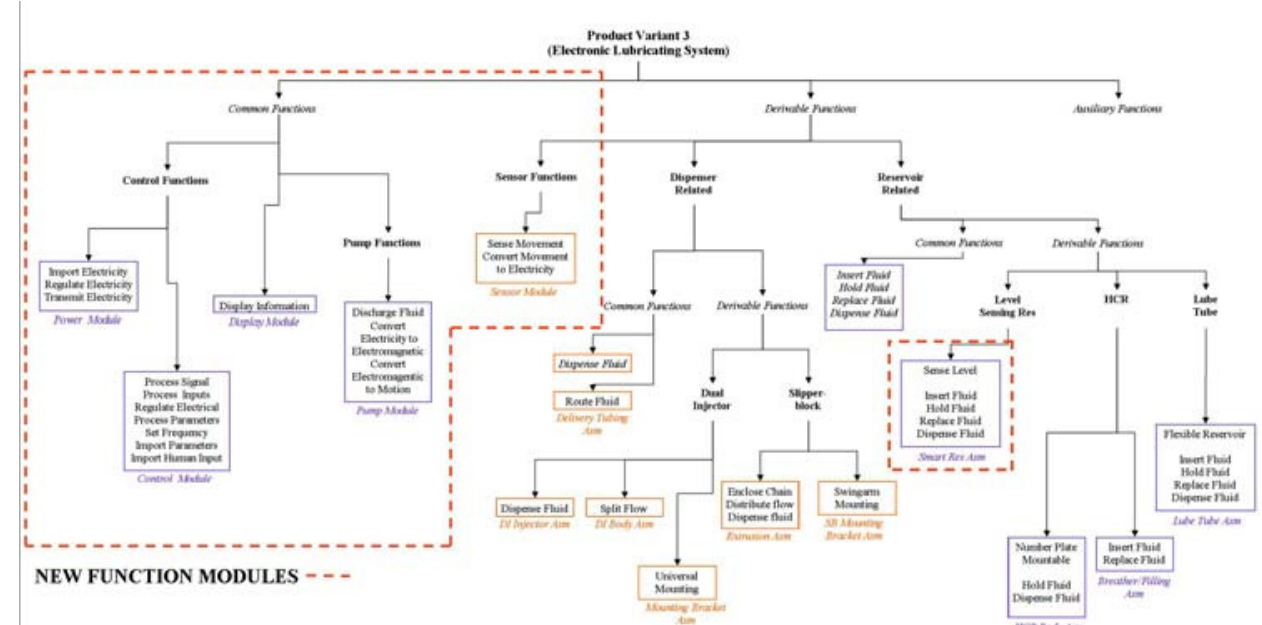

Figure 4 - A Generic Product Family Function Structure (GPFS) highlighting the new modules to be developed.

\section{SME Case Study}

The GeMoCURE methodology has been implemented, in various forms, within large mulinational companies, but the focus of this research is how it can be implemented within a UK-based SME. The SME in question is a manufacturer of chain oiling systems that are marketed as after-market maintenance devices. They have a small product portfolio of around 8 products but are keen to expand this by using their current knowledge, modules and parts to create a new product family. As such, they were prime candidates to make use of this technology and to aid in its development and implementation. The GeMoCURE methodology was implemented into this company with the purpose of introducing modularity concepts that can be used in the design of product families and in module re-use.

The company specialise in chain lubrication and their current methods use manual pressure and vacuum as the activation methods. The company are developing a new electronic system of activation and want to implement this across a whole product family. 


\section{GeMoCURE modular design methodology}

In order to ensure that the system is implemented fully, a new design methodology is created for the company that integrates all the elements of the GeMoCURE process. A summary of how the GeMoCURE methods are used to create a new product family can be seen in Figure 5.

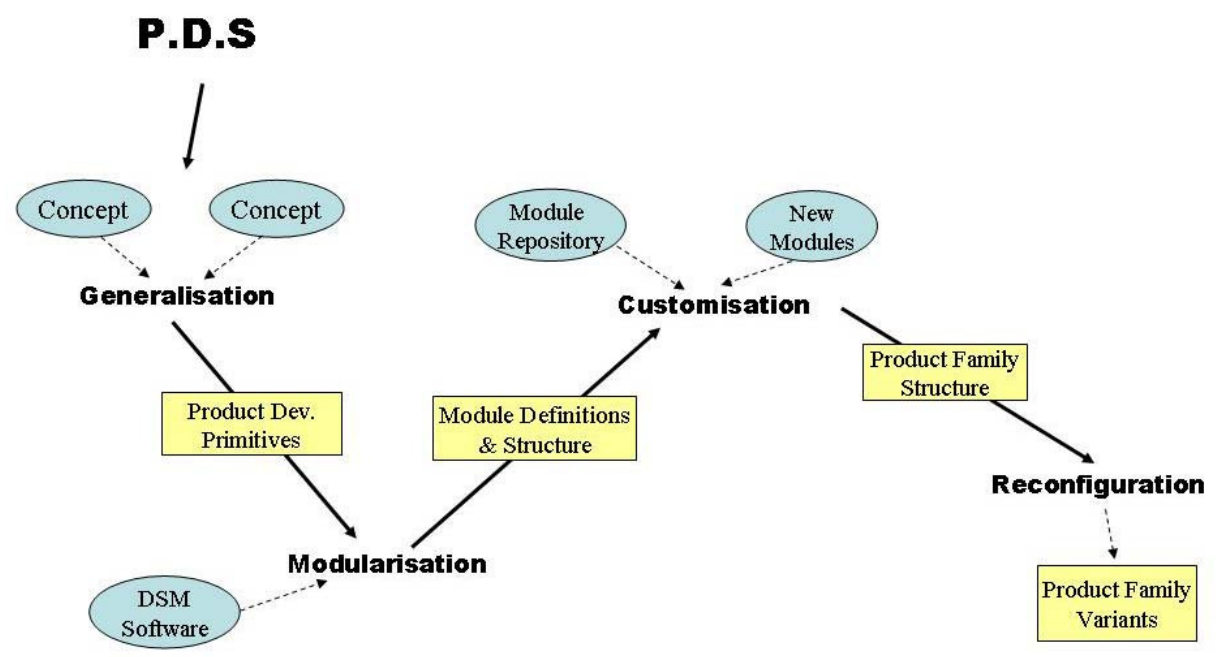

Figure 5 - The process that is carried out to use the GeMoCURE methodology in the creation of new product families.

\subsection{Generalisation / modularisation}

As the company is new to this process it has a large range of legacy components and sub-assemblies that have not been classified into optimised 'modules'. The first stage therefore is to analyse these components and identify how they are currently used in the products and how they can most easily form modules. A generalisation activity can then be carried out for each product and the functions and structural elements can be assigned to these modules. While these won't be optimised or meet the modular definition that has been set down it does describe them in the GeMoCURE language and therefore allow for them to be re-used in new development projects. As this was a small company with few products this was not a strenuous task but this could become prohibitive for larger companies or larger product ranges. To aid in the recording and searching of these modules a database was created that stored the modules and allowed for searching to take place using specific function and structure PDPs. The database also allowed for the linking of files so that the associated documents and product knowledge for each module are always easily available. 


\subsection{Customisation}

For the new product development the first stage is to carry out the 'Generalisation' of the product family. The first step is for the functional generalisation model to be created that shows how the energy, material and information flow through the proposed product and are affected by certain product functions. This model is based on that proposed by Stone et al [2000] and efficiently breaks down the product into meaningful primitives and details the relationships and interdependencies between them. This model can be seen in Figure 6. This model forms the basis of the new product family by defining it in terms of its PDPs i.e. in a language which the previous modules, etc. are described.

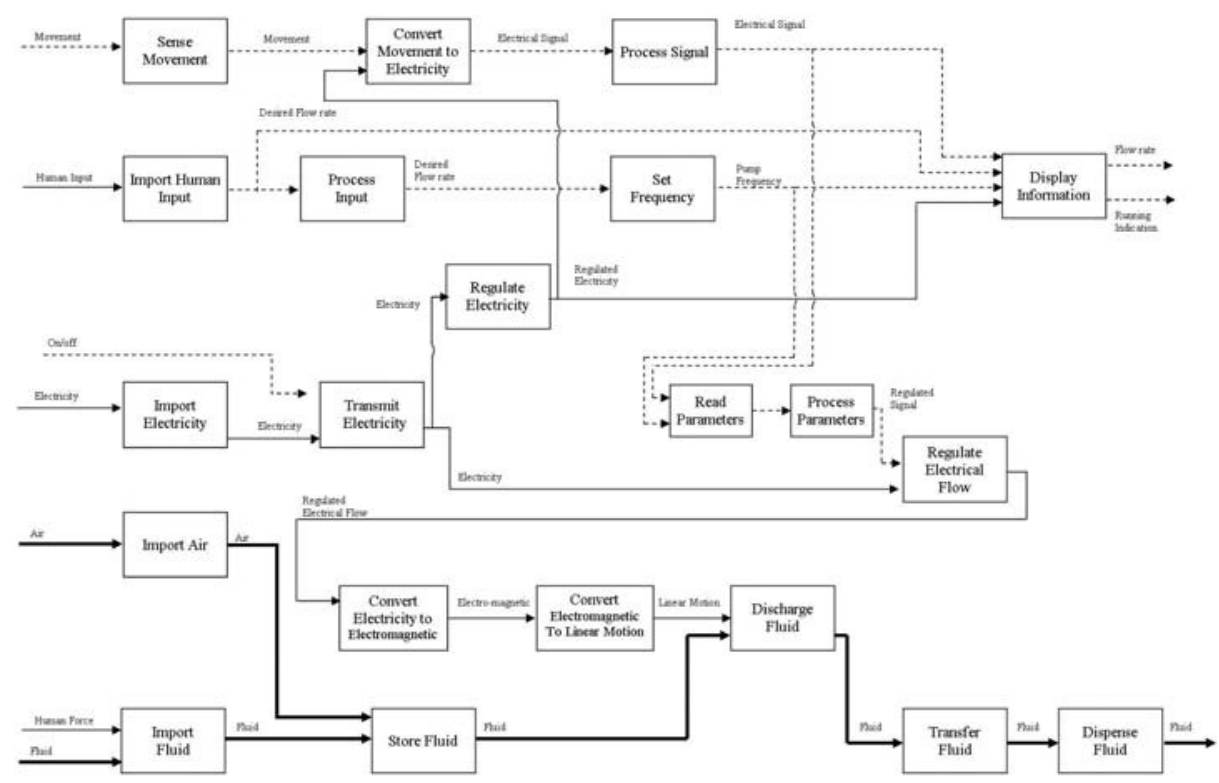

Figure 6 - The function model used to generalise the new proposed product family.

The next stage is to take these new PDPs from the previous section and analyse them to find optimal module structure for the product family. The Dependency Structure Matrix (DSM) software can be applied to the models to optimise primitives into an optimal modular structure. By entering these into the matrix the genetic algorithm is used to cluster the primitives using the dependencies that were defined in the generalisation stage. For this product, the functional model is clustered to produce a series of proposed modules and sub-modules that can be selected by using the MIM. The colour coding of the MIM provides a guide to the strength of the modules and from this suitable clusters of primitives can be classified as proposed modules (see Figure 7). Strongly coloured relationships can be grouped into core modules while relationships that are less strongly coloured can be classed as sub-modules that include a series of stronger modules. 


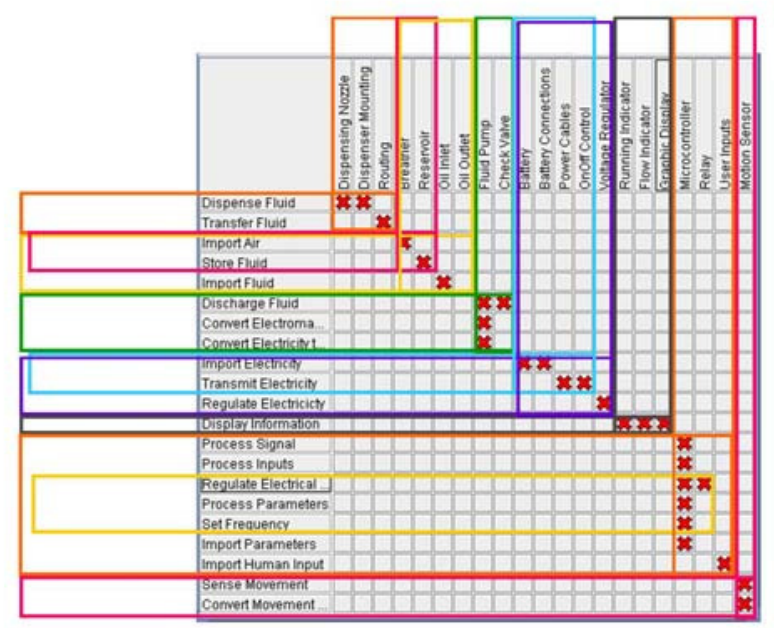

Figure 7 - The Module Identification Module (MIM) for a cross viewpoint matrix showing the identified modules and sub-modules.

The customisation of the new family starts at this stage where a functional comparison is carried out between the required functional modules of the new family and the depository of functional modules present within current company products. The depository is searchable by function so that any current modules that match the requirement of the new product will be flagged up and the designer can then ascertain whether this module is suitable for the new product. To aid in this decision making process the depository contains all the attribute information for each module, the interfacial definitions and any associated knowledge (i.e. materials, manufacturing process, assembly definitions, CAD, etc.). For this new product the designer identified several current modules within the depository that accurately met some functional requirements of the new family. For a specified sub-function, there exists no module that meets the definitions and therefore new modules had to be designed. This is not uncommon as this new product is quite different from the company's current products. These new modules were designed with the functional intent being considered at all times. At this stage it was also important to consider the interfaces that will be present within the new family. By reusing current modules, their interfaces are fixed therefore the new modules have to be designed with the correct interfaces to effectively integrate with these modules. An example of how this was carried out can be seen in Figure 8. This figure shows how a current module is incorporated with an existing module and how the new module was designed with a matching inner circular interface to allow for easy integration and installation. The new module was developed to meet all the functional requirements that were defined so that it can help be part of this product but is also sufficiently independent that it can be used in other new products further in the future. 


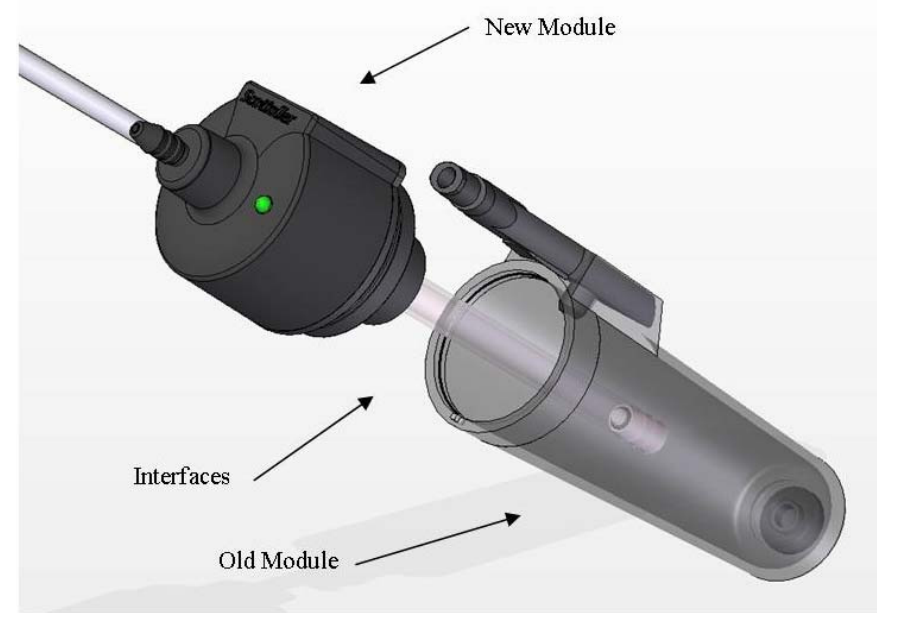

Figure 8 - An example of two modules (one new and one old) from the new product family.

\subsection{Product family reconfiguration}

In most cases there will be more than one functional module that can meet certain requirements of the new product and there will be some requirements that are not essential for the product to function to some degree. In this case this range of modules become classed as derivable modules and form new sections of the product family. By using these derivable modules in different configurations with the product platform different product variations can be created that allow for many products to be brought to market for each product family. Again as the modules are added to the depository, all information and knowledge that has been generated is linked in as well and this knowledge helps make informed decisions over what configurations are suitable and how realistic they are.

For the new company product the modules that were identified along with the new modules that have been created are analysed and a GPFS is created for this new product family - see Figure 4. This GPFS shows that there are four common modules that come together to form the new product platform and that there were six different types of derivable modules identified. When combined with the common functional modules (the product platform) up to eighteen possibly different variations can be created.

From this family, with its eighteen variations, the reconfiguration stage can then be carried out where the customer requirements and market needs can be taken into account and the best configuration (or configurations) can be chosen to be taken to market. One ideal configuration was chosen for this new product to act as a core product but several of the other modules will be sold as add-on products that allow for the functionality to be changed in small ways. This is only possible as the interfaces for these add-ons are matched accurately to the new and old modules that were defined in the product family.

Previous singular product development projects within the company have taken upwards of 18 months to complete but in this case a new product family (with a possible 18 product variations) is scheduled to be completed in around 12 months. This can be attributed to the fact that a large portion of the product family makes use of proven 


\section{GeMoCURE modular design methodology}

modules that are already established within the company. The fact that so many of the modules are proven, reliable modules not only allows for rapid configuration of these new variants and fast time to market but should also guarantee a high level of product quality due to the knowledge and experience already attached to the reused modules.

\subsection{Interface Analysis}

When carrying out modular design - with a view to creating product families and with using design for re-use principles - it is necessary to create good definitions of how the modules interact with one another. It is especially important to have well defined and standardised interfaces between the common modules (the product platform) and the derivable modules [Sundgren (1999)]. Sellgren and Andersson (1998) define an interface as a "pair of mating faces between two elements". In this case this can be expanded to mean the pair of mating features or faces between two modules. In order to cope with the variety of different products they may end up in, the interfaces on modules have to be designed robustly and should preferably be defined early in the design process [Blackenfelt and Sellgren (2000)].

The importance of defining the interfaces within modules is clear and this has been particularly evident within this SME. When modules are designed to work over several product variants and several product families it is important that there are definitions set down as to how these should be handled. In the methodology an initial stage has been added called 'Interface Identification'. The purpose of this stage is to look at both the functional and structural modules and assess the interactions between other modules. In the current company portfolio this is a simple task as the designs are in place and products are in manufacture. Therefore it is simply a case of documenting these and adding them into the depository. By also including interfaces in the modules definitions it gives a better idea of how the modules can actually fit together. It is also critical when designing new function modules, as it is imperative that any new modules that are produced are compatible with the product family. This way when the re-used modules have been defined there will be a definitive list of the interfaces that are present and it will be possible to design new modules to integrate with the product platform.

\section{Future Work and Conclusions}

The focus of the research so far has been implementing the system within an SME and observing how it handles such an environment. This has shown some clear areas that are required to be improved in the system. The first area is the identification of commonality with the function modules and structural modules as at present this is done intuitively by the designers. There is work being carried out in parallel with this project looking at introducing algorithms into these stages to ensure that the identification is carried out optimally. The second area is the implementation of a more formal interface strategy within the methodology. It has been realised how important this is to the overall feasibility of a module re-use strategy and this will be the focus of future research. The primary aim will be to establish standard interface descriptions and allow these to be modelled into the methodology along with the crucial interface attributes. 
This paper has demonstrated a new design methodology aimed at aiding designers in producing modular products and families. By using a series of tools and methods a systematic approach to modular design can be achieved that allows for opportunities for module re-use and rapid product configuration which can lead to reduced product lead times and lower development costs. The methodology consists of four distinct stages; Generalisation, Modularisation, Customisation and Reconfiguration. These four stages were described and shown how the system uses a DSM tool to find the optimal product structure. This structure was then used to sort the company's products into a range of useable modules and a definition of their product families. The system can also be used to create new product variants from these product families by using a system of differentiable modules that can be altered and added to product platforms to create variants.

One of the main outcomes of the implementation of this methodology has been the need for a standardised system of interfaces to allow for effective module re-use. If modules are to be used in many different product variants it is essential that a standard system of interfaces is devised that will allow for this to be carried out efficiently. This has been pinpointed as a key topic for future work and will be built upon within the methodology to introduce a standard set of interfaces.

\section{References}

Baxter D, Gao J, Case K, Harding J, Young B, Cochrane S, Dani S; (2007) 'An engineering design knowledge reuse methodology using process modelling'. Research in Engineering Design, Vol. 18, pp. 37-48.

Thyssen J. and Hansen P.K. (2001) 'Impacts for Modularisation', Proceedings of International Conference on Engineering Design, ICED '01, Glasgow, pp. 547-554.

Gershenson J.K., Prasad G.J. and Zhang Y. (2004) 'Product modularity: measures and design methods'. Journal of Engineering Design, Vol. 15, pp.33-51.

Synopsys Inc. Who can afford a $\$ 193$ Million Chip? (1999) Synopsys Design Reuse Cost Model.

Duffy A.H.B. and Ferns A.F. (1999) 'An Analysis of Design Reuse Benefits', Proceedings of the International Conference on Engineering Design, ICED '99, Munich.

Smith J.S. (2002) 'Multi-Viewpoint Modular Design Methodology', Doctoral Thesis, University of Strathclyde, Glasgow.

Yan X.T., Stewart B., Wang W., Tramscheck R., Liggat J., Duffy A.H.B., Whitfield I., (2007) 'Developing and applying an integrated modular design methodology within an SME', Proceedings of International Design Conference on Engineering Design, ICED '07, Paris.

Wang W.D., Qin X.S., Yan X.T., Tong S.R., Sha Q.Y. (2007) 'Developing a Systematic Method for Constructing the Function Platform of Product Family', IEEE International Conference on Industrial Engineering and Engineering Management (IEEM2007), Singapore.

Jiao J.X., Simpson T. W., Siddique Z. (2006), 'Product Family Design and Platform-Based Product Development: A State-of-the-Art Review', Special Issue on Product Family Design and Platform-Based Product Development, Journal of Intelligent Manufacturing, pp.1-36.

Pahl G. and Beitz P (1994) Engineering Design: A Systematic Approach, (Springer-Verlag Berlin and Heidelberg $\mathrm{GmbH} \& \mathrm{Co}$ ).

Ulrich K.T. and Eppinger S.D. (2003) Product Design and Development, Third Edition (McGraw Hill).

Stone R., Wood K. and Crawford R. (2000) 'A Heuristic method for identifying modules for product architectures'. Design Studies Vol. 21, pp.5-31. 


\section{GeMoCURE modular design methodology}

Gershenson J.K., Prasad G.J., Zhang Y. (2003) 'Product Modularity: definitions and benefits'. Journal of Engineering Design, Vol. 14 , pp. 295-313.

Smith J.S, Duffy A.H.B. (2001) 'Modularity in Support of Design for Re-Use', Proceedings of International Conference on Engineering Design, ICED '01, Glasgow, 195-206

Huang C., Kusiak A. (1998) 'Modularity in Design of Products and Systems', IEEE Transactions on systems, man and cybernetics, 28:66-77.

Sosale S., Hashiemian M. and Gu P. (1997) 'Product Modularisation for Re-use and Recycling', ASME, Design Engineering Division, 94:195-206.

Hofer A.P., Gruenenfelder M. (2001) 'Product Family Management Based on Platform Concepts', Proceeding of International Conference on Engineering Design, Glasgow, 491498.

Schellhammer W., Karandikar H. (2001) 'Metrics for Executing a Product Platform Strategy', Proceeding of International Conference on Engineering Design, Glasgow, 531-538.

Wie M. V., Bryant C., Bohm M. R., Mcadams D. A., Stone R. B. (2005) 'A Model of FunctionalBased Representation'. Artificial Intelligence for Engineering Design, Analysis and Manufacturing, Vol. 19, pp.9-111.

Robertson, D., Ulrich, K. (1998) 'Planning for Product Platforms', Sloan Management Review.

Sundgren N. (1999) 'Introducing Interface Management in New Product Family Development', Journal of Product Innovation Management, 16:40-51.

Sellgren, U., Andersson, K. (1998) 'MOSAIC - a Framework and a Methodology for Behavior Modeling of Complex Systems', Proceeding of Produktmodeller'98, 119-137.

Blackenfelt M., Sellgren U. (2000), 'Design of Robust Interfaces in Modular Products', Proceedings of 2000 ASME Design Engineering Technical Conference, Baltimore, Maryland. 